\title{
Binocular Vision in Patients with Multiple Sclerosis
}

This article was published in the following Dove Press journal:

Clinical Optometry

\author{
Amparo Gil-Casas ${ }^{1,2}$ \\ David P Piñero-Llorens ${ }^{2}$ \\ Ainhoa Molina-Martin (1D) \\ 'Clínica Optométrica, Fundació Lluís \\ Alcanyís, University of Valencia, Valencia, \\ Spain; ${ }^{2}$ Optics and Visual Perception \\ Group (GOPV), Department of Optics, \\ Pharmacology, and Anatomy, University \\ of Alicante, Alicante, Spain
}

Purpose: Oculomotor disorders have been reported in multiple sclerosis (MS) in up to $80 \%$ of cases. There have been studies evaluating binocular vision in several neurological diseases, but not in MS. Considering that a high percentage of eye-movement anomalies have been reported, the aim of this study was to analyze binocular vision in these subjects. Methods: A total of 59 participants with MS -21 with monocular optic neuritis, eleven with binocular optic neuritis, and 27 without optic neuritis - and 26 age-matched controls were enrolled. Binocular vision was analyzed using near point of convergence (NPC), positive and negative fusional vergence for far and near distance, measurement of heterophoria at both distances with cover and modified Thorington tests, and random-dot stereoscopy.

Results: The percentage of subjects with abnormal NPC values was highest in the MS group, followed by the MSONm (MS with optic neuritis in one eye), MSONb (MS with optic neuritis in both eyes), and control groups. MS patients showed an esophoric trend at near distance. Positive fusional vergence showed no significant differences between control and MS groups, but higher variability in recovery was found in MS groups. Negative fusional vergence at near distance showed significant differences between the control group and the two MS groups, with optic neuritis for both break-point and recovery values. A high percentage of patients with MS had alterations on stereopsis.

Conclusion: Alterations in binocular vision were present in MS, with divergence at near distance and stereopsis the most affected parameters. Likewise, MS patients with optic neuritis showed worse binocular vision.

Keywords: multiple sclerosis, binocular vision, near point of convergence, heterophoria, fusional vergences, stereopsis

\section{Introduction}

Multiple sclerosis (MS) is defined as a neurodegenerative central nervous system disease associated with the presence of inflammatory localized lesions causing myelin loss. ${ }^{1-3}$ It is more prevalent in women than in men, and produces disability in young adults behind traumatic lesions.

The visual system is severely affected by MS, and some visual symptoms have been found to be associated with these patients, such as affected of lowcontrast visual acuity, visual field, or color perception. ${ }^{4-6}$ Optic neuritis (ON) is a common disorder associated with these patients, and is present in up to $50 \%$ of subjects with MS during the course of the disease. ${ }^{7}$ Most vision-related symptoms in MS can be attributed to present or resolved ON in this population, but some of these findings are present even if no $\mathrm{ON}$ has been suffered. ${ }^{8}$ Therefore, ophthalmological and optometric evaluation is essential in the followup of patients with MS.
Correspondence: Ainhoa Molina-Martin Department of Optics, Pharmacology, and Anatomy, University of Alicante,

Carretera San Vicente del Raspeig, San Vicente del Raspeig, 03690, Alicante, Spain

Tel +34965903-400

Email ainhoa.molina@ua.es 
Localized lesions of MS can involve a large part of the visual pathway, and may lead to problems in oculomotor activity. ${ }^{9}$ Oculomotor disorders, such as saccadic dysmetria, impaired smooth pursuit, nystagmus, and impaired convergence, ${ }^{10-12}$ have been reported in MS in up to $80 \%$ of cases. These alterations can appear as the first symptom of the disease, but can can show up in relapse and even in the course of the MS. ${ }^{13}$ Moreover, these alterations in oculomotricity have been associated with high levels of disability, with a negative impact on quality of life. ${ }^{14}$ Likewise, fatigue, defined as a sense of exhaustion, lack of energy, or tiredness, is present in at least $75 \%$ of patients, ${ }^{15}$ with potential involvement of extraocular muscles and consequently the possibility of generating alterations in oculomotricity.

There have been studies evaluating oculomotricity and binocular vision in several neurological diseases, such as Parkinson's, spinocerebellar ataxia, Huntington's, multiple-system atrophy and Alzheimer's, ${ }^{16,17}$ but not in MS. A high percentage of eye-movement anomalies have been reported in patients with MS due to the demyelination process of the neural substrate of ocular muscles, and affected binocular vision could be another visual consequence of the MS, as occurs in other neurodegenerative diseases. Due to the scarce scientific literature in this regard, the aim of this study was to explore binocular vision in patients with MS and report the presence of abnormal results in comparison to healthy controls.

\section{Methods}

\section{Subjects}

Subjects were recruited from two clinical centers: the Optometric Clinic of the Lluis Alcanyis Foundation (University of Valencia, Valencia, Spain) and the Optometric Clinic of the University of Alicante (Alicante, Spain). All participants signed a written informed consent prior to inclusion in the study and after being informed about its nature according to the tenets of the Declaration of Helsinki. The protocol was approved by the ethics committees of the University of Alicante (UA-2018-03-02) and University of Valencia (H1527574656645).

Patients with MS were included regardless of duration of disease, age at onset, or type of MS. Subjects had been previously diagnosed and followed by their neurologists and were recruited from local associations. Patients with ocular disorders before the diagnosis of MS were excluded, but a high percentage had had resolved ON during the course of the disease. Patients with MS, both with and without previous ON, were included, but subdivided into three groups: MS group (MS without ON), MSONm (MS with ON in one eye), and MSONb (MS with $\mathrm{ON}$ in both eyes).

Although subjects had no strabismic alterations and thus had a priori presence of binocular vision (almost simultaneous vision), they were not exempt from suffering nonstrabismic alterations on functionality (eg, asymptomatic reduced near fusional vergence). Likewise, the presence of nonstrabismic binocular alterations in the MS groups could have been due to normal prevalence and not caused by the disease. Since diagnosis of nonstrabismic binocular vision alterations depends on the criteria selected and there is no agreement on normal values for studied parameters in the presbyopic population, ${ }^{26}$ the results obtained in MS groups were compared with those obtained in an age- and sex-matched control group to evaluate prevalence in relative terms.

The control group comprised on healthy subjects (without any active ocular pathology) and without strabismus or amblyopia (without strabismic binocular vision alterations) that had been enrolled randomly as a reference population. Subjects with refractive errors wore their best corrected-refraction spectacles and were measured with compensation for distance/near vision. Binocular examination was evaluated following the order described in the next section.

\section{Procedure}

\section{Near Point of Convergence}

Near point of convergence (NPC) without accommodative stimulus was measured in all subjects. ${ }^{18}$ A sharpened tip of a pencil was placed $40-50 \mathrm{~cm}$ from the patient's eyes in the primary gaze, and then the examiner slowly moved the target toward the patient's eyes. The patient was instructed to report when the target appeared double or split into two. At this point, the examiner stopped moving the pencil and measured the distance from the target to the patient's eye in centimeters, and this was the NPC break point. If the patient did not report double vision, but the examiner saw that one eye had lost fixation and deviated, that position was considered the NPC break point. The examiner then moved the target away along the same path until single vision was reported and this distance was also measured, corresponding to the NPC-recovery point. Difference between break and recovery distance, defined as NPCrecovery range, was also calculated and averaged. 


\section{Horizontal Heterophoria}

To evaluate ocular alignment and detect the presence of horizontal heterophoria, two free-space methods were considered: the cover test ${ }^{19}$ (objective method in which the examiner assesses the presence and level of misalignment) and the modified Thorington test ${ }^{20}$ (subjective method based on patient-reported perception). The cover-uncover test was carried out to determine if there was strabismus, whereas the alternating cover test was used to quantify the magnitude of the deviation. This measurement was performed for far and near distance $(4 \mathrm{~m}$ and $40 \mathrm{~cm}$, respectively). The modified Thorington test was performed by positioning a red Maddox rod over the right eye and asking the patient about the number at which the red line crossed while he/she was fixating on a light stimulus arranged in the center of the card. This measurement was performed for far and near distance $(2 \mathrm{~m}$ and $40 \mathrm{~cm}$, respectively). Both tests were conducted on primary gaze position. Positive values represented esophoria and negative values exophoria.

\section{Fusional Vergence}

Positive and negative fusional vergence ${ }^{21}$ were measured using a horizontal prism bar (Gulden B-16, horizontal $1 \Delta-45 \Delta$ ) and a handheld fixation target containing a single vertical column of letters of $20 / 32$ visual acuity for far and near distance $(4 \mathrm{~m}$ and $40 \mathrm{~cm}$, respectively) Each subject was asked to report when the letters became double (split into two). This was defined as the break value. Then, prismatic demand was reduced until single vision was restored. This was defined as the recovery value. Negative fusional vergence was measured firstly to avoid excessive stimulation of convergence in recovery from positive fusional vergence measures. ${ }^{22}$ Differences between break and recovery values, defined as recovering ranges, were also calculated and averaged.

\section{Stereopsis}

Stereopsis was measured using the Randot preschool stereoacuity test (Stereo Optical, Chicago, IL, USA). ${ }^{23}$ This test was administered and scored (from 40 to 800 arc seconds) according to the manufacturer's instructions. If stereopsis were immeasurable, the Worth four-dot test ${ }^{24}$ in a darkened room was used to assess if the subject were able to see five dots, confirming the perception of diplopia, or two red or three green dots, indicating the presence of suppression in one eye. For analytical purposes, results were recorded as proposed by Webber et $\mathrm{al}^{25}$ and binocular function obtained as the logarithmic value of the threshold obtained in seconds of arc. For example, a stereopsis value of 200 arc seconds was scored as $\log _{200}=2.3$. A fixed score of four was assigned when diplopia was present, and a score of five in cases of suppression.

\section{Statistical Analysis}

Statistical analyses were performed using SPSS 26.0.0. The Shapiro-Wilk test revealed no parameters followed normal distribution, so nonparametric tests were applied. Differences between groups (control, MS, MSONm, and $\mathrm{MSONb}$ ) were analyzed with the Kruskal-Wallis test for NPC (break point, recovery point, and range), cover test, modified Thorington test, PFV, and NFV (break point, recovery point, and range), and stereopsis (expressed as logarithmic value). For parameters showing differences between groups, additional Mann-Whitney paired analysis (with Bonferroni adjustment) was done. Three measurements were obtained from every studied parameter, and the average value was analyzed. During the acquisition of some parameters, subjects with MS seemed to be more variable on their responses. Intrasubject standard deviation of PFV and NFV was analyzed to represent the variability of the three consecutive measures.

Although participants had no strabismic binocular alterations, they were not exempt from suffering accommodative and nonstrabismic binocular dysfunction, ie, we could not assume that subjects were binocularly normal, so all groups were compared with normal values provided in the literature and the percentage of subjects with abnormal values in each group compared. There is no agreement on diagnostic criteria for binocular dysfunction in the scientific literature, ${ }^{26}$ but our purpose was not diagnosis but comparison between groups, so a specific criterion for each studied parameter was chosen. The percentage of eyes with exophoria, orthophoria, or esophoria measured by the cover and modified Thorington tests is also reported for all groups.

\section{Results}

A total of 59 subjects with MS and 26 age-matched healthy subjects were recruited: 27 in the MS group, 21 in the MSONm group, and eleven in the MSONb group. From the initial sample of 68 subjects, nine were excluded from analysis of NPC, horizontal heterophoria, PFV, and $\mathrm{NFV}$, due to the presence of suppression in one eye (one in the MS group, five in the MSONm group, and three in the 
MSONb group). All patients were considered for analysis of stereopsis outcomes, since the scoring system used ${ }^{20}$ allowed the inclusion of patients without measurable stereopsis values.

The mean age of subjects was $49 \pm 10$ years ( 20 women and seven men) in the MS group, $50 \pm 9$ years (13 women and six men) in the MSONm group, $50 \pm 7$ years (nine women and two men) in the MSONb group, and $50 \pm 8$ years (16 women and ten men) in the control group. There were no statistically significant differences among groups regarding age $(p=0.82)$ or sex $(p=0.63)$. Mean refractive errors per group (spherical equivalent) were $-0.30 \pm 1.31$ $\mathrm{D}$ in the control group, $-0.29 \pm 2.76 \mathrm{D}$ in the MS group, $0.32 \pm 1.94 \mathrm{D}$ in the MSONm group, and $0.17 \pm 2.03 \mathrm{D}$ in the MSONb group $(p=0.42)$. All participants in both control and study groups achieved a good corrected logMAR visual acuity with no statistically significant differences among groups ( $p=0.31$ ): $-0.08 \pm 0.06$ in the control group, $-0.03 \pm 0.08$ in the MS group, $0.02 \pm 0.11$ in the MSONm group, and $0.08 \pm 0.12$ in the MSONb group. Means $\pm \mathrm{SD}$ obtained for the parameters evaluated (ranges and intrasubject SD) are summarized in Table 1. Abnormal results in each group are summarized in Table 2. Likewise, the percentage of eyes according to type of horizontal heterophoria is summarized in Table 3.

\section{Near Point of Convergence}

Higher values for break point, recovery point, and range of NPC were found in the MSONm group than the MS and MSONb groups, the latter two with fairly similar values. The control group showed the best NPC results(Table 1). Although differences between groups did not reach statistical significance for break or recovery points ( $p=0.61$ and $p=0.15$, respectively), differences among groups in the range were close to significance $(p=0.06)$.

To determine the percentage of subjects with abnormal NPC values, our results were compared to those of Scheiman et $\mathrm{al}^{18}$ and NPC break and recovery values of $5 \mathrm{~cm}$ and $7 \mathrm{~cm}$, respectively, considered. The proportion of subjects with abnormal values on both break and recovery points (Table 2) was highest in the MS group (48\%), followed by the MSONm (42\%) and MSONb (36\%) groups. The control group had the lowest proportion of subjects with abnormal NPC results (26\%).

\section{Horizontal Heterophoria}

For far distance, both the cover and modified Thorington tests showed values close to orthophoria in the control,
MS, and MSONb groups, whereas a trend toward esophoria was found in the MSONm group (Table 1). For near distance, the cover test showed exophoric values for the control, MS, and MSONb groups, whereas a trend toward esophoria was found in the MSONm group. The modified Thorington test showed exophoric values for the control and MSONb groups, orthophoria for the MS group, and esophoria for MSONm group. Differences in heterophoria measurements with tests for far and near distance among groups did not reach statistical significance.

To determine the percentage of subjects with abnormal heterophoria, our results were compared to those of CantóCerdán et $\mathrm{al}^{19}$ for the cover test and Lyon et $\mathrm{al}^{20}$ for the modified Thorington test. For far distance, normal values of $-1 \pm 2 \Delta$ and $0 \pm 2 \Delta$ were considered, respectively. For near distance, normal values of $-3 \pm 4 \Delta$ and $-1 \pm 4 \Delta$ were considered, respectively. Results for subjects with abnormal horizontal heterophoria for far and near distance on both cover and modified Thorington tests are shown in Table 2.

Regarding the type of horizontal heterophoria reported (Table 3), percentages differed slightly between the cover and Thorington tests, due to differences in the grade of dissociation of both tests. In general, for both far and near distance, more esophoria was obtained in sclerosis groups than the control group.

\section{Fusional Vergence}

Positive fusional vergence measurements for far distance showed the highest values for both break and recovery points in the control group, whereas the MSONb group achieved the lowest values, as can be seen in Table 1. The ranges for far distance were similar in all groups, although the MSONb group showed a trend toward smaller ranges. No statistically significant differences were found between groups $(p>0.05)$. Nevertheless, intrasubject SD corresponding to break and recovery points differed significantly between the control and MS groups $(p<0.05)$. Positive fusional vergence measurements for near distance followed the same trend, with better values in the control group, but not reaching statistically significant differences between groups $(p>0.05)$. Intrasubject SD corresponding to break and recovery points differed significantly when comparing MSONm and MS groups with the control group $(p<0.05)$.

Negative fusional vergence measurements for far distance showed the highest values for both break and recovery points 
Table I Means \pm SD and Medians (IQR) of Parameters Analyzed in the Four Groups of Patients Evaluated Those Comparisons Obtaining Statistical Significance are Marked with an Asterisk. Exophoria was Included with Negative Sign and Esophoria with Positive. Sw: Intrasubject SD from Three Consecutive Measurements

\begin{tabular}{|c|c|c|c|c|c|c|c|}
\hline & & & Control & MS & MSONm & MSONb & $\mathbf{p}$ \\
\hline \multirow[t]{6}{*}{ Near point of convergence $(\mathrm{cm})$} & \multirow{2}{*}{\multicolumn{2}{|c|}{ Break }} & $3.60 \pm 3.74$ & $5.20 \pm 4.4 I$ & $6.11 \pm 7.06$ & $5.31 \pm 6.53$ & \multirow[t]{2}{*}{0.61} \\
\hline & & & $4.00(6.25)$ & $6.00(7.50)$ & $4.00(8.00)$ & $4.50(6.00)$ & \\
\hline & \multirow{2}{*}{\multicolumn{2}{|c|}{ Recovery }} & $5.38 \pm 5.4 \mathrm{I}$ & $9.85 \pm 8.32$ & $10.81 \pm 10.68$ & $8.45 \pm 7.95$ & \multirow[t]{2}{*}{0.15} \\
\hline & & & $6.00(10.25)$ & $10.00(15.00)$ & $8.50(15.00)$ & $10.00(10.50)$ & \\
\hline & \multirow{2}{*}{\multicolumn{2}{|c|}{ Range }} & $1.76 \pm 1.96$ & $4.64 \pm 4.92$ & $4.70 \pm 5.10$ & $3.13 \pm 2.42$ & \multirow[t]{2}{*}{0.06} \\
\hline & & & $1.50(7.00)$ & $4.00(6.00)$ & $3.00(7.50)$ & $3.00(10.00)$ & \\
\hline \multirow[t]{4}{*}{ Cover test $(\Delta)$} & \multirow{2}{*}{\multicolumn{2}{|c|}{ Distance }} & $-0.24 \pm 0.66$ & $0.32 \pm 1.24$ & $1.44 \pm 4.44$ & $0.10 \pm 2.07$ & \multirow[t]{2}{*}{0.39} \\
\hline & & & $0(2.00)$ & $0(0.00)$ & $0(1.00)$ & $0(3.00)$ & \\
\hline & \multirow{2}{*}{\multicolumn{2}{|c|}{ Near }} & $-2.88 \pm 4.62$ & $-2.59 \pm 6.72$ & $0.27 \pm 7.69$ & $-2.82 \pm 7.27$ & \multirow[t]{2}{*}{0.70} \\
\hline & & & $-4.00(8.00)$ & $-4.00(10.00)$ & $-2.50(11.00)$ & $-3.00(7.00)$ & \\
\hline \multirow[t]{4}{*}{ Modified Thorington test $(\Delta)$} & \multirow{2}{*}{\multicolumn{2}{|c|}{ Distance }} & $0.12 \pm 0.75$ & $0.40 \pm 2.76$ & $1.63 \pm 5.10$ & $-0.30 \pm 4.11$ & \multirow[t]{2}{*}{0.62} \\
\hline & & & $0(4.00)$ & $0(1.50)$ & $0(4.30)$ & $0(3.10)$ & \\
\hline & \multirow{2}{*}{\multicolumn{2}{|c|}{ Near }} & $-2.18 \pm 3.21$ & $0.00 \pm 7.58$ & $1.50 \pm 5.19$ & $-1.50 \pm 6.16$ & \multirow[t]{2}{*}{0.12} \\
\hline & & & $-3.00(4.50)$ & $-1.00(7.00)$ & $0(6.50)$ & $-0,50(7.00)$ & \\
\hline \multirow[t]{10}{*}{ Positive fusional vergence $(\Delta)$} & \multirow[t]{10}{*}{ Distance } & Break & $18.74 \pm 5.94$ & $16.73 \pm 8.22$ & $17.50 \pm 7.40$ & $14.90 \pm 8.57$ & \multirow[t]{2}{*}{0.38} \\
\hline & & & $17.33(11.00)$ & $14.66(15.00)$ & $19.33(13.67)$ & $14.00(24.00)$ & \\
\hline & & Sw & $0.84 \pm 1.46$ & $1.93 \pm 2.34$ & $3.52 \pm 3.32$ & $2.54 \pm 1.97$ & \multirow[t]{2}{*}{$0.002^{*}$} \\
\hline & & & $0(1.15)$ & $1.15(3.06)$ & $2.89(3.58)$ & $2.00(3.85)$ & \\
\hline & & Recovery & $11.92 \pm 4.53$ & $10.83 \pm 5.43$ & $11.51 \pm 5.59$ & $10.09 \pm 6.21$ & \multirow[t]{2}{*}{0.67} \\
\hline & & & $11.00(7.33)$ & $8.66(16.00)$ & $13.33(9.33)$ & $9.33(10.67)$ & \\
\hline & & Sw & $0.48 \pm 0.66$ & $1.93 \pm 1.63$ & $2.84 \pm 2.90$ & $1.70 \pm 1.41$ & \multirow[t]{2}{*}{$<0.00 I^{*}$} \\
\hline & & & $0(1.15)$ & $2.00(3.06)$ & $2.00(3.01)$ & $1.15(1.16)$ & \\
\hline & & Range & $6.82 \pm 3.70$ & $5.77 \pm 4.10$ & $6.14 \pm 2.98$ & $4.8 \mathrm{I} \pm 3.44$ & \multirow[t]{2}{*}{0.26} \\
\hline & & & $6.66(6.00)$ & $4.00(7.00)$ & $5.33(4.67)$ & $3.66(4.33)$ & \\
\hline & \multirow[t]{8}{*}{ Near } & Break & $23.73 \pm 9.87$ & $19.64 \pm 11.18$ & $23.00 \pm 7.86$ & $|7.69 \pm 9.9|$ & \multirow[t]{2}{*}{0.24} \\
\hline & & & $23.33(16.17)$ & $18.66(18.67)$ & $22.00(11.67)$ & I4.66 (20.00) & \\
\hline & & Sw & $1.23 \pm 1.25$ & $3.67 \pm 4.49$ & $3.57 \pm 2.47$ & $1.32 \pm 1.40$ & \multirow[t]{2}{*}{$0.002 *$} \\
\hline & & & 1.15 (2.89) & $2.89(5.03)$ & $2.89(2.98)$ & 1.15 (2.89) & \\
\hline & & Recovery & $|8.9| \pm 8.7 \mid$ & $15.20 \pm 10.29$ & $15.82 \pm 6.82$ & $12.03 \pm 9.67$ & \multirow[t]{2}{*}{0.28} \\
\hline & & & $18.00(13.00)$ & $14.00(14.33)$ & $14.66(11.67)$ & $10.66(8.00)$ & \\
\hline & & Sw & $0.68 \pm 0.96$ & $2.48 \pm 1.93$ & $2.98 \pm 1.78$ & $0.83 \pm 0.94$ & \multirow[t]{2}{*}{$<0.00 I^{*}$} \\
\hline & & & $0(1.15)$ & $2.3 \mathrm{I}(2.85)$ & $2.89(2.04)$ & $1.00(1.73)$ & \\
\hline
\end{tabular}

(Continued) 
Table I (Continued).

\begin{tabular}{|c|c|c|c|c|c|c|c|}
\hline & & & Control & MS & MSONm & MSONb & $\mathbf{p}$ \\
\hline & & \multirow[t]{2}{*}{ Range } & $4.80 \pm 3.80$ & $4.43 \pm 4.29$ & $7.17 \pm 4.53$ & $5.66 \pm 2.24$ & \multirow[t]{2}{*}{0.06} \\
\hline & & & $4.50(3.25)$ & $4.00(2.33)$ & $6.33(6.67)$ & $5.00(1.67)$ & \\
\hline \multirow[t]{20}{*}{ Negative fusional vergence $(\Delta)$} & \multirow[t]{10}{*}{ Distance } & \multirow[t]{2}{*}{ Break } & $7.43 \pm 2.36$ & $6.15 \pm 2.14$ & $7.12 \pm 3.40$ & $6.54 \pm 3.55$ & \multirow[t]{2}{*}{0.15} \\
\hline & & & $7.33(3.33)$ & $6.00(2.83)$ & $6.00(3.33)$ & $5.33(4.00)$ & \\
\hline & & \multirow[t]{2}{*}{ Sw } & $0.48 \pm 0.57$ & $0.89 \pm 0.74$ & $1.08 \pm 0.99$ & $0.73 \pm 0.58$ & \multirow[t]{2}{*}{$0.02 *$} \\
\hline & & & $0(1.15)$ & $1.15(1.15)$ & $1.15(1.15)$ & $1.15(1.15)$ & \\
\hline & & \multirow[t]{2}{*}{ Recovery } & $5.17 \pm 2.32$ & $3.43 \pm 1.6 \mathrm{I}$ & $3.64 \pm 2.04$ & $2.69 \pm 1.44$ & \multirow[t]{2}{*}{$0.003^{*}$} \\
\hline & & & $4.00(7.33)$ & $3.33(2.00)$ & $3.66(8.67)$ & $2.00(2.33)$ & \\
\hline & & \multirow[t]{2}{*}{ Sw } & $0.22 \pm 0.46$ & $0.50 \pm 0.54$ & $\mathrm{I} .0 \mathrm{I} \pm 2.0 \mathrm{I}$ & $0.57 \pm 0.5 \mathrm{I}$ & \multirow[t]{2}{*}{$<0.00 I^{*}$} \\
\hline & & & $0(1.15)$ & $0.29(1.15)$ & $0(1.15)$ & $0.58(1.15)$ & \\
\hline & & \multirow[t]{2}{*}{ Range } & $2.25 \pm 1.33$ & $2.7 I \pm 1.99$ & $3.48 \pm 1.85$ & $3.84 \pm 3.59$ & \multirow[t]{2}{*}{$0.01 *$} \\
\hline & & & $2.00(1.50)$ & $2.00(0.75)$ & $2.66(0.17)$ & $3.00(1.67)$ & \\
\hline & \multirow[t]{10}{*}{ Near } & \multirow[t]{2}{*}{ Break } & $|3.46 \pm 3.8|$ & $10.81 \pm 2.82$ & $10.10 \pm 2.83$ & $9.21 \pm 3.73$ & \multirow[t]{2}{*}{$0.007^{*}$} \\
\hline & & & $13.33(6.17)$ & $10.66(5.33)$ & $10.00(2.67)$ & $10.00(8.00)$ & \\
\hline & & \multirow[t]{2}{*}{ Sw } & $0.53 \pm 0.58$ & $1.27 \pm 0.87$ & $2.03 \pm 2.61$ & $1.29 \pm 0.90$ & \multirow[t]{2}{*}{$0.002 *$} \\
\hline & & & $0(1.15)$ & I.I5 (0.85) & 1.15 (1.91) & I.I5 (0.85) & \\
\hline & & \multirow[t]{2}{*}{ Recovery } & $10.58 \pm 3.70$ & $7.91 \pm 2.80$ & $6.19 \pm 3.29$ & $6.03 \pm 3.04$ & \multirow[t]{2}{*}{$0.00 I^{*}$} \\
\hline & & & $10.00(4.67)$ & $8.00(5.33)$ & $6.66(5.33)$ & $5.33(5.33)$ & \\
\hline & & \multirow[t]{2}{*}{ Sw } & $0.44 \pm 0.57$ & $1.15 \pm 1.13$ & $1.61 \pm 1.12$ & $1.39 \pm 0.72$ & \multirow[t]{2}{*}{$<0.001 *$} \\
\hline & & & $0(1.15)$ & 1.15 (1.15) & $1.15(1.16)$ & $1.15(0.85)$ & \\
\hline & & \multirow[t]{2}{*}{ Range } & $2.87 \pm 1.78$ & $2.90 \pm 1.08$ & $3.91 \pm 1.85$ & $3.18 \pm 1.55$ & \multirow[t]{2}{*}{0.10} \\
\hline & & & $2.33(1.50)$ & $2.66(1.33)$ & $3.33(3.33)$ & $2.66(2.00)$ & \\
\hline \multirow[t]{2}{*}{ Log stereopsis } & \multirow{2}{*}{\multicolumn{2}{|c|}{ Near }} & $1.70 \pm 0.12$ & $2.09 \pm 0.64$ & $2.59 \pm 1.20$ & $3.04 \pm 1.21$ & \multirow[t]{2}{*}{$<0.001 *$} \\
\hline & & & $1.69(0.18)$ & $2.00(0.70)$ & $2.00(0.99)$ & $2.30(2.00)$ & \\
\hline
\end{tabular}

in the control group. Only the recovery point showed statistically significant differences between the three sclerosis groups and the control group ( $p<0.05$ in all cases). Intrasubject SD showed a statistically significant difference between the MS and MSONm groups and the control group $(p<0.05)$. Negative fusional vergence measurements for near distance showed significant differences between the control group and the two groups with $\mathrm{ON}$ for both break and recovery points $(p<0.05)$. These results are summarized in Figure 1. Although there were no significant differences between the MS and control groups in near-distance break and recovery points $(p>0.05)$, intrasubject SD differed significantly $(p<0.05)$. No statistically significant differences between groups were found in the negative fusional vergence range $(p>0.05)$.

To determine the percentage of subjects with abnormal positive and negative fusional vergence values, our results were compared to those of Lyon et al, ${ }^{20}$ and normal values of $12 \pm 7 \Delta$ (break point) and $7 \pm 5 \Delta$ (recovery point) were considered for positive fusional vergence, and of $8 \pm 4 \Delta$ (break point) and $5 \pm 3 \Delta$ (recovery point) for negative fusional vergence for far distance. For near distance, normal values of $20 \pm 11 \Delta$ and $14 \pm 8 \Delta$ were considered for positive fusional vergence and $13 \pm 6 \Delta$ (break point) and 9 $\pm 4 \Delta$ (recovery point) for negative fusional vergence. 
Table 2 Binocular Abnormalities per Group Classified According to the Normative Values Described in the text. For Cover and Modified Thorington Tests Results Displayed Separately According to the Type of Heterophoria

\begin{tabular}{|c|c|c|c|c|c|c|}
\hline & & & Control, n=26 & MS, $n=27$ & MSONm, $n=19$ & MSONb, $n=I I$ \\
\hline \multicolumn{3}{|l|}{ Near point of convergence } & 7 (26\%) & $13(48 \%)$ & $8(42 \%)$ & $4(36 \%)$ \\
\hline \multirow[t]{4}{*}{ Cover test } & \multirow{2}{*}{\multicolumn{2}{|c|}{ Far }} & 3 (1 I.5\%) & $4(16 \%)$ & 7 (35\%) & $4(36.3 \%)$ \\
\hline & & & -3 exo & 3 eso 1 exo & 4 eso 3 exo & 3 eso I exo \\
\hline & \multirow{2}{*}{\multicolumn{2}{|c|}{ Near }} & $6(23 \%)$ & 14 (53\%) & 9 (45\%) & $3(28 \%)$ \\
\hline & & & 6 eso & 8 eso 6 exo & 6 eso 3 exo & 3 eso \\
\hline \multirow[t]{4}{*}{ Modified Thorington test } & \multirow{2}{*}{\multicolumn{2}{|c|}{ Far }} & I (3.8\%) & $9(34.6 \%)$ & $9(45 \%)$ & 5 (45.4\%) \\
\hline & & & I eso- & 6 eso 3 exo & 7 eso 2 exo & 3 eso 2 exo \\
\hline & \multirow{2}{*}{\multicolumn{2}{|c|}{ Near }} & 5 (19.2\%) & $8(30 \%)$ & $6(33.3 \%)$ & $4(40 \%)$ \\
\hline & & & 3 eso 2 exo & 5 eso 3 exo & 6 eso & 3 eso I exo \\
\hline \multirow[t]{4}{*}{ Positive fusional vergence } & \multirow[t]{2}{*}{ Far } & Break & $4(15 \%)$ & 12 (44\%) & $12(52 \%)$ & $5(45.5 \%)$ \\
\hline & & Recovery & I (3.8\%) & $3(11 \%)$ & I (5\%) & $3(27 \%)$ \\
\hline & \multirow[t]{2}{*}{ Near } & Break & 5 (19.2\%) & II (40.4\%) & $4(2 \mid \%)$ & $5(45.4 \%)$ \\
\hline & & Recovery & $2(7.6 \%)$ & $4(14.8 \%)$ & I (5.6\%) & $4(36.3 \%)$ \\
\hline \multirow[t]{4}{*}{ Negative fusional vergence } & \multirow[t]{2}{*}{ Far } & Break & $4(15 \%)$ & $10(37 \%)$ & $6(31.5 \%)$ & $6(54.5 \%)$ \\
\hline & & Recovery & 0 & $5(19 \%)$ & $3(16 \%)$ & $4(36 \%)$ \\
\hline & \multirow[t]{2}{*}{ Near } & Break & 21 (80\%) & 27 (100\%) & 19 (100\%) & II (100\%) \\
\hline & & Recovery & 3 (1 I.5\%) & 10 (38.4\%) & $10(52.6 \%)$ & 8 (72.7\%) \\
\hline \multicolumn{3}{|l|}{ Stereopsis } & 2 (7.7\%) & 15 (53\%) & 17 (70.8\%) & II (100\%) \\
\hline
\end{tabular}

Percentages of subjects with abnormal values for far and near distance on both positive and negative fusional vergence are shown in Table 2.

\section{Stereopsis}

Higher scores, indicating worst binocularity, were obtained in the MSONb group, followed by the MSONm and MS groups. The best stereopsis values were found in the control group, as shown in Table 1. There were significant differences between the control group and the three sclerosis groups $(p<0.02$ in all cases). Likewise, the MS group showed statistically significant differences in stereopsis when compared with the $\mathrm{MSONb}$ group $(p=0.02)$, but not with the MSONm group ( $p>0.05)$. Statistically significant differences were also observed between the two groups with $\mathrm{ON}$, as can be seen in Figure 2.

According to previous studies on healthy adults, ${ }^{27}$ an upper limit of 70 seconds of arc is considered normal. The percentage of subjects with abnormal stereoscopic values was highest in the MSONb group (100\%), followed by the MSONm group (70.8\%) and the MS group (53\%). In the control group, only $7.7 \%$ of subjects showed an abnormal stereopsis value. Stereopsis was correlated with the magnitude of near-distance heterophoria in the MS group ( $r=$ $-0.57, p=0.002$ ).

\section{Discussion}

Neurodegenerative disorders affect the central nervous system, often leading to motor and sensorial problems. Eye-movement control is complex, and involves many brain areas that can be affected by demyelination, disturbing the normal functioning of binocularity. ${ }^{9}$ Studies have reported alterations in vergence, saccades, and smooth pursuit in some demyelinating pathologies, such as Parkinson's disease, spinocerebellar ataxia, Huntington's disease, and multiple-system atrophy. ${ }^{16,17}$ However, although eye-movement disorders in MS have been widely 
Table 3 Distribution of Heterophoria Measurements Obtained Using the Cover Test and Modified Thorington Test

\begin{tabular}{|c|c|c|c|c|c|c|}
\hline & & & Control, n=26 & MS, $n=27$ & MSONm, n=19 & MSONb, $n=11$ \\
\hline \multirow[t]{6}{*}{ Cover test } & Far & Orthophoria & $22(88 \%)$ & $20(74 \%)$ & $10(53 \%)$ & $4(40 \%)$ \\
\hline & & Exophoria & $3(12 \%)$ & $2(7.5 \%)$ & $4(21 \%)$ & $4(40 \%)$ \\
\hline & & Esophoria & - & $5(18.5 \%)$ & $5(26 \%)$ & $3(20 \%)$ \\
\hline & Near & Orthophoria & $2(7 \%)$ & $2(7.7 \%)$ & $2(10.5 \%)$ & - \\
\hline & & Exophoria & 18 (70\%) & 17 (6I.5\%) & II (58\%) & $8(73 \%)$ \\
\hline & & Esophoria & $6(23 \%)$ & $8(30 \%)$ & $6(3 \mid .5 \%)$ & $3(27 \%)$ \\
\hline \multirow[t]{6}{*}{ Modified Thorington test } & Far & Orthophoria & 14 (54\%) & $8(30.76 \%)$ & 7 (37\%) & $3(27 \%)$ \\
\hline & & Exophoria & $5(19 \%)$ & $10(38.46 \%)$ & $3(16 \%)$ & $3(27 \%)$ \\
\hline & & Esophoria & $7(27 \%)$ & $9(30.76 \%)$ & $9(47 \%)$ & $5(46 \%)$ \\
\hline & Near & Orthophoria & $2(7.7 \%)$ & $6(23 \%)$ & 6 (3I.5\%) & $2(18 \%)$ \\
\hline & & Exophoria & $19(73 \%)$ & $14(50 \%)$ & $6(31.5 \%)$ & $5(46 \%)$ \\
\hline & & Esophoria & 5 (19.3\%) & 7 (27\%) & 7 (37\%) & $4(36 \%)$ \\
\hline
\end{tabular}

described in the literature, ${ }^{3,8-10,12,13}$ there have been no studies to date on NPC, horizontal heterophoria, and fusional vergence values in MS patients.

An association between $\mathrm{ON}$ and $\mathrm{MS}$ has been widely described by other authors, with most patients suffering a decrease in visual pathways, ${ }^{10-13}$ but there have been no studies analyzing specifically the impact of such decreases on binocular vision. The current study reports binocular vision parameters in patients with MS with and without $\mathrm{ON}$ and compares these results with those obtained in healthy subjects. The percentage of eyes with alterations was also reported and compared between groups, considering the normative data from previous peer-reviewed literature. The final aim was to confirm if binocular vision was altered

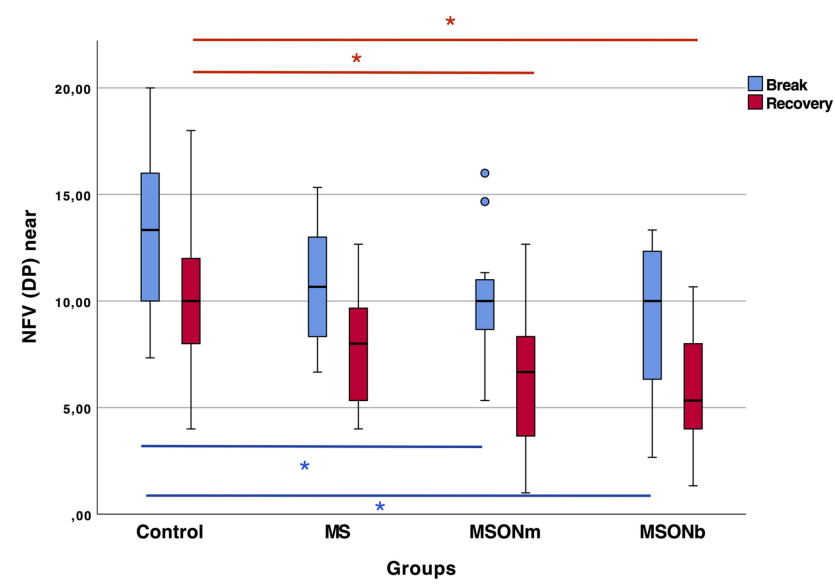

Figure I Break and recovery values for negative fusional vergence at near distance for all groups in prismatic diopters. Asterisks denote statistically significant differences between groups. more in subjects with MS than controls and if this alteration were more pronounced in patients with monocular or binocular ON.

Based on the results of the current series, MS does not strictly seem to affect NPC, since no differences were present between the studied groups, but there was a higher trend in patients with MS, specifically in those patients suffering $\mathrm{ON}$ in one eye. Also, better results in recovery ranges were found in controls than study groups. These results suggest that once convergence is decompensated (double vision), MS patients find it harder to recover single vision. This situation seemed to be more persistent in subjects with binocular ON, and even more so in subjects with monocular ON. In the present study, a reference value was selected according to a previous study, ${ }^{18}$ but any other criteria could have been used to classify results as normal or not. This criterion was quite strict, since narrower NPC values are considered normal when compared with other studies. ${ }^{18,26}$ The percentage of control subjects showing abnormal results in the current sample was $26 \%$, which is high, but it was even higher when considering MS subjects (36\%-48\%). Regarding the methodology, although measurement with an accommodative stimulus might be a more appropriate test to determine NPC for diagnotic purposes, in the current sample all subjects were presbyopes and measured with their best correction for near vision, and thus the involvement of accommodation in the measurement seems to be quite limited.

Based on the data obtained in the current study, no significant differences were found between groups, with control subjects showing results consistent with previous studies evaluating heterophoria in healthy subjects. ${ }^{19,20}$ 


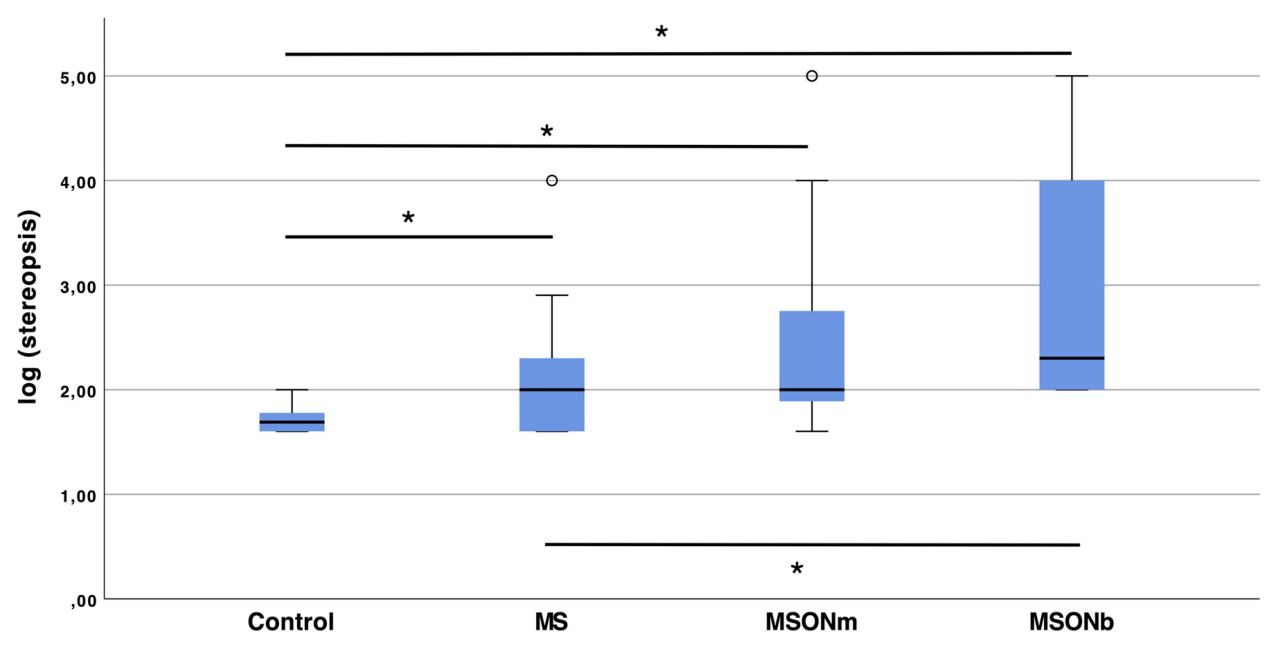

Figure 2 Stereoacuity in log arc seconds for all groups. Asterisks denote statistically significant differences between groups.

However, a trend toward more esophoric results was found in patients with MS, specifically in those with monocular $\mathrm{ON}$, for near vision, and more markedly on the modified Thorington test. The percentage of subjects with abnormal heterophoria was higher in MS groups, showing higher proportions of esophoria than normal subjects. Although there have been no previous studies evaluating the impact of $\mathrm{ON}$ on heterophorias, the results of the current series suggest that patients with $\mathrm{ON}$ tend to be more esophoric than healthy subjects.

Although the cover test ${ }^{28}$ and modified Thorington test ${ }^{29}$ have demonstrated good replicability and no statistically significant differences have been reported by other authors, these tests cannot be interchangeable. ${ }^{30}$ Our results showed that values obtained with the Thorington test were more esophoric for far and near distance than the cover test, as has been reported before by other authors with healthy subjects, ${ }^{31}$ perhaps due to the different level of dissociation achieved with each method. Future studies should be conducted to determine exactly how measurement methodology affects the diagnosis of binocular anomalies in subjects with MS.

The results of the current study suggest that no differences should be expected for positive fusional vergence in MS patients compared to healthy subjects in terms of break point, recovery point, or range. However, higher intrasubject variability was found, perhaps caused by patient fatigue ${ }^{15}$ when measuring vergence several consecutive times or instability of fixation reported in MS patients. ${ }^{8,32,33}$ Regarding divergence fusional ranges, the current study found that divergence was affected in all subjects with MS, specifically in those with ON in one or both eyes. This is consistent with results obtained by other authors reporting significant latency in divergence in other neurodegenerative diseases, such as Parkinson's, that maintained a convergence fusional range similar to controls ${ }^{34}$ Furthermore, pontine lesions causing weakness of divergence are usually present in demyelinating diseases. ${ }^{35}$ In the current series, although no correlation was found between divergence fusional range and neardistance heterophoria, more esophoria was found at near distance in MS patients, suggesting that alterations in divergence can be frequent in these subjects.

Deficits in stereoscopic vision in MS may be due to alterations in ocular movements, but also to demyelination during the disease. ${ }^{9}$ In the current study, stereopsis was lower in all MS patients, but even more so in those with ON (especially binocular ON cases). Stereoscopic deficiency in MS was consistent with previous studies, even when asymptomatic ${ }^{36}$ and no ON were present, ${ }^{37}$ but also stereoscopic deficiency has been found to be associated with optic nerve disease. ${ }^{38}$ Since no correlations were found between stereopsis and NPC, horizontal heterophoria, or fusional vergence and no significant differences found in healthy subjects on some binocular parameters, we suggest that stereoscopic reduction is caused more by damage in visual pathways than in oculomotor control centers, but future studies should be conducted to confirm these results.

\section{Conclusion}

Negative fusional vergence at near distance and stereopsis seem to be the binocular vision parameters affected most by MS, becoming worse in patients also suffering ON. In 
addition, the high proportion of esophoria in MS patients indicates that more studies are needed for analyzing the heterophoria-divergence relationship in these subjects. Although NPC and positive fusional vergence did not differ significantly between MS and healthy subjects, the variability of such parameters suggests a certain level of instability that should be investigated.

\section{Funding}

David P Piñero was supported by the Ministry of Economy, Industry, and Competitiveness of Spain within the program Ramón y Cajal, RYC-2016-20471.

\section{Disclosure}

The authors report no conflicts of interest in this work.

\section{References}

1. Lublin FD, Reingold SC, Cohen JA, et al. Defining the clinical course of multiple sclerosis: the 2013 revisions. Neurology. 2014;83 (3):278-286. doi:10.1212/WNL.0000000000000560

2. Pozzilli C, Tomassini V, Marinelli F, Paolillo A, Gasperini C, Bastianello S. "Gender gap" in multiple sclerosis: magnetic resonance imaging evidence. Eur $J$ Neurol. 2003;10(1):95-97. doi:10.1046/j.1468-1331.2003.00519.x

3. Serra A, Chisari CG, Matta M. Eye movement abnormalities in multiple sclerosis: pathogenesis, modeling, and treatment. Front Neurol. 2018;9(FEB):31. doi:10.3389/fneur.2018.00031

4. Galetta KM, Balcer LJ. Measures of visual pathway structure and function in MS: clinical usefulness and role for MS trials. Mult Scler Relat Disord. 2013;2(3):172-182. doi:10.1016/j.msard.2012.12.004

5. Villoslada P, Cuneo A, Gelfand J, Hauser SL, Green A. Color vision is strongly associated with retinal thinning in multiple sclerosis. Mult Scler J. 2012;18(7):991-999. doi:10.1177/1352458511431972

6. Cheng H, Laron M, Schiffman JS, Tang RA, Frishman LJ. The relationship between visual field and retinal nerve fiber layer measurements in patients with multiple sclerosis. Investig Ophthalmol Vis Sci. 2007;48(12):5798-5805. doi:10.1167/iovs.07-0738

7. Chan JW. Optic neuritis in multiple sclerosis. Ocul Immunol Inflamm. 2002;10(3):161-186. doi:10.1076/ocii.10.3.161.15603

8. Gil-Casas A, Piñero Llorens DP, Molina-Martin A. Ocular fixation and macular integrity by microperimetry in multiple sclerosis. Graefe's Arch Clin Exp Ophthalmol. 2020.

9. Gil-Casas A, Piñero DP, Molina-Martin A. Binocular, accommodative and oculomotor alterations in multiple sclerosis: a review. Semin Ophthalmol. 2020;35(2):103-115. doi:10.1080/08820538.2020.174 4671

10. Graves J, Balcer LJ. Eye disorders in patients with multiple sclerosis: natural history and management. Clin Ophthalmol. 2010;4 (1):1409-1422. doi:10.2147/OPTH.S6383

11. Frohman EM, Frohman TC, Zee DS, McColl R, Galetta S. The neuro-ophthalmology of multiple sclerosis. Lancet Neurol. 2005;4 (2):111-121. doi:10.1016/S1474-4422(05)00992-0

12. Reulen JPH, Sanders EACM, Hogenhuis LAH. Eye movement disorders in multiple sclerosis and optic neuritis. Brain. 1983;106 (1):121-140. doi:10.1093/brain/106.1.121

13. Niestroy A, Rucker JC, Leigh RJ. Neuro-ophthalmologic aspects of multiple sclerosis: using eye movements as a clinical and experimental tool. Clin Ophthalmol. 2007;1(3):267-272.
14. Kes VB, Čengić L, Cesarik M, et al. Quality of life in patients with multiple sclerosis. Acta Clin Croat. 2013;52(1):107-112.

15. Braley TJ, Chervin RD. Fatigue in multiple sclerosis: mechanisms, evaluation, and treatment. Sleep. 2010;33(8):1061-1067. doi:10.10 93/sleep/33.8.1061

16. Kang SL, Shaikh AG, Ghasia FF. Vergence and strabismus in neurodegenerative disorders. Front Neurol. 2018;9(MAY):1. doi:10.33 89/fneur.2018.00299

17. Anderson TJ, MacAskill MR. Eye movements in patients with neurodegenerative disorders. Nat Rev Neurol. 2013;9(2):74-85. doi:10. 1038/nrneurol.2012.273

18. Scheiman M, Gallaway M, Frantz KA, et al. Nearpoint of convergence: test procedure, target selection, and normative data. Optom Vis Sci. 2003;80(3):214-225. doi:10.1097/00006324-200303000-00011

19. Cantó-Cerdán M, Cacho-Martínez P, García-Muñoz Á. Measuring the heterophoria: agreement between two methods in non-presbyopic and presbyopic patients. J Optom. 2018;11(3):153-159. doi:10.1016/j. optom.2017.10.002

20. Lyon DW, Goss DA, Horner D, Downey JP, Rainey B. Normative data for modified Thorington phorias and prism bar vergences from the Benton-IU study. Optometry. 2005;76(10):593-599. doi:10.1016/ j.optm.2005.08.014

21. Lança CC, Rowe FJ. Measurement of fusional vergence: a systematic review. Strabismus. 2019;27(2):88-113. doi:10.1080/09273972.20 19.1583675

22. McDaniel C, Fogt N. Vergence adaptation in clinical vergence testing. Optometry. 2010;81(9):469-475. doi:10.1016/j.optm.2010. 01.012

23. Birch E, Williams C, Drover J, et al. Randot ${ }^{\circledR}$ preschool stereoacuity test: normative data and validity. J AAPOS. 2008;12(1):23-26. doi:10.1016/j.jaapos.2007.06.003

24. Roper-Hall G. The "Worth" of the worth four dot test. Am Orthopt J. 2004;54(1):112-119. doi:10.3368/aoj.54.1.112

25. Webber AL, Wood JM, Thompson B, Birch EE. From suppression to stereoacuity: a composite binocular function score for clinical research. Ophthalmic Physiol Opt. 2019;39(1):53-62. doi:10.1111/ opo. 12599

26. Cacho-Martínez P, García-Muñoz Á, Ruiz-Cantero MT. Is there any evidence for the validity of diagnostic criteria used for accommodative and nonstrabismic binocular dysfunctions? J Optom. 2014;7 (1):2-21. doi:10.1016/j.optom.2013.01.004

27. Piano MEF, Tidbury LP, O'Connor AR. Normative values for near and distance clinical tests of stereoacuity. Strabismus. 2016;24 (4):169-172. doi:10.1080/09273972.2016.1242636

28. Johns HA, Manny RE, Fern K, Hu YS. The intraexaminer and interexaminer repeatability of the alternate cover test using different prism neutralization endpoints. Optom Vis Sci. 2004;81 (12):939-946.

29. Cebrian JL, Antona B, Barrio A, Gonzalez E, Gutierrez A, Sanchez I. Repeatability of the modified thorington card used to measure far heterophoria. Optom Vis Sci. 2014;91(7):786-792. doi:10.1097/ OPX.0000000000000297

30. Antona B, Gonzalez E, Barrio A, Barra F, Sanchez I, Cebrian JL. Strabometry precision: intra-examiner repeatability and agreement in measuring the magnitude of the angle of latent binocular ocular deviations (heterophorias or latent strabismus). Binocul Vis Strabolog Q. 2011;26(2):91-104.

31. Sanker N, Prabhu A, Ray A. A comparison of near-dissociated heterophoria tests in free space. Clin Exp Optom. 2012;95 (6):638-642. doi:10.1111/j.1444-0938.2012.00785.x

32. Nij Bijvank JA, Petzold A, Coric D, et al. Quantification of visual fixation in multiple sclerosis. Investig Ophthalmol Vis Sci. 2019;60 (5):1372-1383. doi:10.1167/iovs.18-26096

33. Mallery RM, Poolman P, Thurtell MJ, et al. Visual fixation instability in multiple sclerosis measured using SLO-OCT. Investig Ophthalmol Vis Sci. 2018;59(1):196-201. doi:10.1167/iovs.17-22391 
34. Hanuška J, Bonnet C, Rusz J, et al. Fast vergence eye movements are disrupted in Parkinson's disease: a video-oculography study. Park Relat Disord. 2015;21(7):797-799. doi:10.1016/j.parkreldis.2015. 04.014

35. Karatas M. Internuclear and supranuclear disorders of eye movements: clinical features and causes. Eur J Neurol. 2009;16(12): 1265-1277. doi:10.1111/j.1468-1331.2009.02779.x

36. Heravian J, Moghaddam AAS, Najjaran M, et al. Visual evoked potentials, short wave-length automated perimetry, standard automated perimetry, contrast sensitivity and stereoacuity testing in visually asymptomatic eyes of patients with multiple sclerosis. Iran J Ophthalmol. 2013;25(1):45-52.
37. Sobaci G, Demirkaya S, Gundogan FC, Mutlu FM. Stereoacuity testing discloses abnormalities in multiple sclerosis without optic neuritis. J Neuroophthalmol. 2009;29(3):197-202. doi:10.1097/ WNO.0b013e3181b56764

38. Friedman JR, Kosmorsky GS, Burde RM. Stereoacuity in patients with optic nerve disease. Arch Ophthalmol. 1985;103(1):37-38. doi:10.1001/archopht.1985.01050010041014

\section{Clinical Optometry}

\section{Publish your work in this journal}

Clinical Optometry is an international, peer-reviewed, open access journal publishing original research, basic science, clinical and epidemiological studies, reviews and evaluations on clinical optometry. All aspects of patient care are addressed within the journal as well as the practice of optometry including economic and business analyses. Basic and clinical research papers are published that cover

Submit your manuscript here: https://www.dovepress.com/clinical-optometry-journal

\section{Dovepress}

all aspects of optics, refraction and its application to the theory and practice of optometry. The manuscript management system is completely online and includes a very quick and fair peer-review system, which is all easy to use. Visit http://www.dovepress.com/ testimonials.php to read real quotes from published authors. 